\title{
Implementación de los principios de distribución en planta en el área de acabados y almacén de una empresa de producción de partes ortopédicas
}

\section{Implementation of plant layout principles in the finishing and warehouse area of an orthopedic parts manufacturing company}

DOI: $10.46932 / \mathrm{sfjdv} 3 \mathrm{n} 1-121$

Received in: January $20^{\text {th }}, 2022$

Accepted in: February $1^{\text {st }}, 2022$

Alejandro Lozano González

Profesor de Ingeniería Industrial en la Universidad de Guadalajara

Institución: Centro Universitario de Ciencias Exactas e Ingenierías

Correio eletrônico: alejandro.lgonzalez@academicos.udg.mx

\section{Luz Karina Hernández Garza}

Profesora de Ingeniería Industrial en la Universidad de Guadalajara

Institución: Centro Universitario de Ciencias Exactas e Ingenierías y empresaria del giro textil

Correio eletrônico: luz.hgarza@academicos.udg.mx

Marco Antonio Serratos Macías

Trabaja en la industria metal mecánica y es profesor suplente de ingeniería industrial Institución: Universidad de Guadalajara en el Centro Universitario de Ciencias Exactas e Ingenierías

Correio eletrônico:marcoser175@hotmail.com

José de Jesús Cabrera Chavarría

Profesor de ingeniería en la Universidad de Guadalajara

Institución: Centro Universitario de Tonalá

Correio eletrônico: jose.cabrera@academicos.udg.mx

Julieta Carrasco García

Profesora de ingeniería en la Universidad de Guadalajara

Institución: Centro Universitario de Tonalá

Correio eletrônico: julieta.cgarcia@ academicos.udg.mx

\section{Roberto Huerta Orozco}

Profesor de ingeniería industria en la Universidad de Guadalajara Institución: Centro Universitario de Ciencias Exactas e Ingenierías Correio eletrônico: roberto.huerta@academicos.udg.mx

\section{RESUMEN}

Este trabajo de investigación está realizado para detallar el proceso de identificación y solución de problemáticas relacionadas a la distribución en planta en una empresa del giro ortopédico y como se puede eficientar los distintos procesos operativos que se llevan a cabo en dos áreas específicas. El análisis de las problemáticas llevó a determinar que las áreas de almacén y acabados de esta empresa necesitaban una nueva distribución que satisficiera las necesidades y requerimientos de la producción, es así, que se concluyó que las herramientas que podrían satisfacer estas necesidades sería la aplicación de los principios de la distribución en planta. Los principios de distribución en planta ayudaron de manera puntual a ordenar los espacios físicos, las herramientas y maquinarias, así como al personal, los cuales habían sufrido 
diversos movimientos en función de la necesidad de producción debido al impacto del crecimiento que ha tenido la empresa razón por la cual había imposibilitado la toma de decisiones justificadas en las necesidades de espacio y distribución. La aplicación de estos principios, dan como resultado un aumento significativo en la productividad de los operarios y las máquinas al reducir los tiempos de traslado y tiempos de búsqueda de materiales y productos.

Palabras clave: distribución, principios, procesos, tiempos, eficiencia, layout y espacio.

\begin{abstract}
This research work is carried out to detail the process of identification and solution of problems related to plant distribution in an orthopedic company and how the different operative processes that are carried out in two specific areas can be made more efficient. The analysis of the problems led to determine that the warehouse and finishing areas of this company needed a new distribution that would meet the needs and requirements of production, thus, it was concluded that the tools that could meet these needs would be the application of the principles of plant layout. The principles of plant layout helped in a timely manner to organize the physical spaces, tools and machinery, as well as personnel, which had undergone various movements according to the need for production due to the impact of the growth of the company, which had made it impossible to make decisions based on the needs of space and distribution. The application of these principles results in a significant increase in the productivity of the operators and machines by reducing transfer times and search times for materials and products.
\end{abstract}

Keywords: distribution, principles, processes, time, efficiency, layout and space.

\title{
1 INTRODUCCIÓN
}

La distribución en planta es la disposición de los equipos, espacios y personal que permiten la correcta y eficiente ejecución de un sistema de producción y no hay empresa alguna que no quiera ser eficiente en sus procesos ya que esa eficiencia se traduce en una disminución de costos y por ende, en un aumento de la utilidad.

Los objetivos de la distribución en planta podemos decir que siempre estarán de manera directa o indirecta dentro de los objetivos de las empresas de producción de bienes ya que siempre se buscará asegurar la eficiencia, eficacia, seguridad y comodidad de los ambientes de trabajo así como el lograr distribución mas económica posible que garantice la ejecución de los procesos y la minimización del manipuleo de los materiales, esos objetivos siempre serán fundamentales y por ese motivo, generarán un área de oportunidad para cualquier empresa en cualquier circunstancia.

La distribución en planta enlista seis principios básicos que garantizarán el cumplimiento de los objetivos ya mencionados y que, de llevarse a cabo de manera puntual, lograrán repercutir favorablemente en la optimización de los procesos.

En el caso de la empresa objeto de estudio, ubicada en la zona metropolitana de Guadalajara, ha presentado un constante crecimiento en el último par de años, lo que ha originado diversas problemáticas de congestión de materiales y falta de espacio en diversas áreas resaltando principalmente el área de 
acabados y el almacén de producto terminado, concentrando en ellas la mayor parte de las problemáticas.

En este proyecto se pretende identificar las causas de las problemáticas e implementar por medio de acciones concretas, los principios de la distribución en planta los cuales son: principio de la integración total, principio de la mínima distancia, principio de recorrido, principio de espacio cúbico, principio de satisfacción y seguridad y principio de flexibilidad.

\section{DESARROLLO}

Se realizó un análisis de Pareto por área para encontrar en qué lugar se encontraban mayores problemas de distribución, por lo que se llevó el registro de incidentes por área durante dos semanas, los incidentes que se tomarían en cuenta para el conteo son: retrasos en ordenes de trabajo, congestión en pasillos, congestión en mesas de trabajo, excesivo movimiento manual de productos en proceso o productos terminados y perdida de material de trabajo.

Los resultados del análisis fueron los siguientes:

Tabla 1. Resultados del análisis de incidencias

\begin{tabular}{|c|c|c|c|}
\hline Areas & Incidencias & Porcentaje & Acumulado \\
\hline Acabados & 21 & $39.62 \%$ & $39.62 \%$ \\
\hline Almacén de PT y PP & 15 & $28.30 \%$ & $67.92 \%$ \\
\hline Centros de Maquinado & 7 & $13.21 \%$ & $81.13 \%$ \\
\hline Tornos & 4 & $7.55 \%$ & $88.68 \%$ \\
\hline Pintura & 4 & $7.55 \%$ & $96.23 \%$ \\
\hline Esmeriles & 2 & $3.77 \%$ & $100.00 \%$ \\
\hline TOTAL & 53 & $100 \%$ & \\
\hline
\end{tabular}

Gráfico 1. Gráfico de Pareto del análisis de incidencias

\section{Análisis de Incidencias}

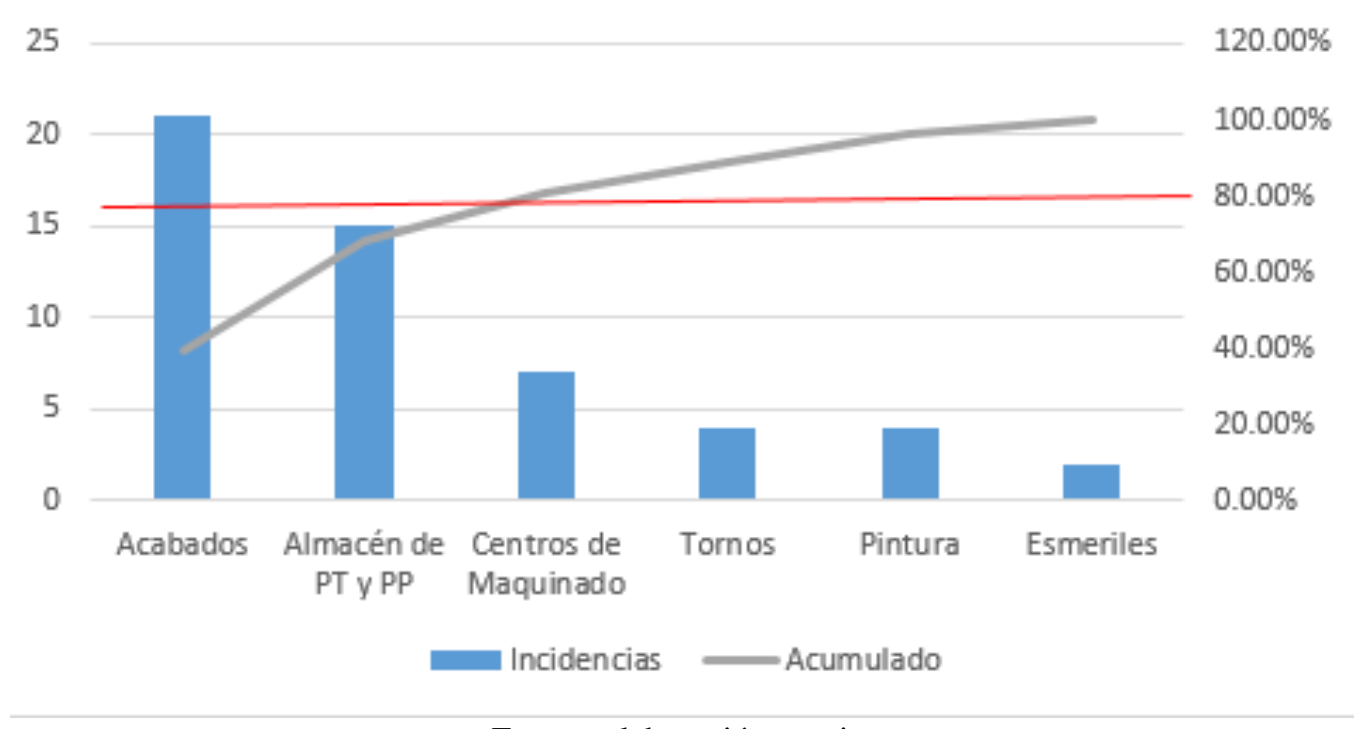

Fuente: elaboración propia. 
Con base en los resultados anteriores se llegó a la conclusión que, resolviendo las problemáticas de distribución en el área de acabados y almacén, se podía dar solución al casi $80 \%$ de las incidencias totales, por lo que se toman como áreas prioritarias, así mismo se llega la conclusión de que es necesario el implementar los principios de la distribución en planta. (Méndez-Mantuano 2022)

La implementación de los principios se desarrolló con una capacitación inicial sobre lo que es la distribución en planta y como esta ayuda a resolver distintas problemáticas en la compañía. La capacitación está pensada para todo el personal operativo de estas áreas y los jefes directos. Se contempló un par de sesiones de 30 minutos donde se mostraría el contexto actual de las áreas, la problemática y el cómo los principios ayudarán en la solución. La capacitación fue llevada a cabo bajo el siguiente esquema y con los siguientes grupos de trabajo:

Tabla 2. Cuadro de dispersión de horarios para la capacitación

\begin{tabular}{|c|c|}
\hline Fechas y Horarios & Integrantes del grupo \\
\hline $\begin{array}{c}\text { Lunes } 21 \text { de junio } 2021 \\
\text { Martes } 23 \text { de junio } 2021 \\
11: 00 \mathrm{hrs}\end{array}$ & $\begin{array}{l}\text { Caciel Ruvalcaba } \\
\text { Juan Carlos Franco } \\
\text { Antonio Acosta } \\
\text { Erick Montes } \\
\text { Antonio Padilla } \\
\text { Mariell Enciso } \\
\text { Jordi Padilla } \\
\text { Alberto Díaz }\end{array}$ \\
\hline $\begin{array}{c}\text { Martes } 22 \text { de junio } \\
\text { Jueves } 23 \text { de junio } \\
11: 00 \mathrm{hrs}\end{array}$ & $\begin{array}{l}\text { Saúl Ortiz } \\
\text { Edwin Ayala } \\
\text { Francisco Díaz } \\
\text { Cesar de la Rosa } \\
\text { Abraham de la Cruz } \\
\text { Rocio Morones } \\
\text { Eder Suazo }\end{array}$ \\
\hline $\begin{array}{c}\text { Martes } 22 \text { de junio } \\
\text { Jueves } 23 \text { de junio } \\
11: 30 \mathrm{hrs}\end{array}$ & $\begin{array}{l}\text { Marisol Santana } \\
\text { Cristina Arellano } \\
\text { Rosy Argumaniz } \\
\text { Ivonne Jiménez } \\
\text { Alexis Ramselle } \\
\text { Mario González } \\
\end{array}$ \\
\hline $\begin{array}{c}\text { Miércoles } 22 \text { de junio } \\
\text { Viernes } 24 \text { de Junio } \\
11: 30 \text { hrs }\end{array}$ & $\begin{array}{l}\text { Marco Rodriguez } \\
\text { Jorge Duarte } \\
\text { Jesús Aceves } \\
\text { Gustavo Rios } \\
\text { Alejandro Negrete } \\
\text { Irving Monroy }\end{array}$ \\
\hline
\end{tabular}

Fuente: elaboración propia.

La capacitación realizada a todo el personal arrojó un balance positivo ya que se encontró sinergia para desarrollar todas las acciones de mejora para optimizar las áreas planteadas como primordiales. Los compañeros integrantes de esas áreas (almacén y acabados) coincidieron puntualmente en la necesidad de mejorar la distribución de las áreas y mostraron su plena disposición para colaborar en ello. Las principales problemáticas que se encontraron en las áreas fueron: 
En almacén:

1. Pasillos congestionados

2. Producto disperso en mesas de trabajo para empaquetado y surtimiento de productos

3. Producto revuelto en racks de almacenamiento

4. Producto sucio

En acabados:

1. Mesas de trabajo con material en espera

2. Herramienta dispersa

3. Insumos dispersos

Al analizar las problemáticas encontradas las podemos empatar con los seis principios de la distribución en planta y confirmamos la hipótesis de cómo estos principios ayudarán en gran medida a disipar las problemáticas.

Las aplicaciones de dichos principios son:

1. Principio de la integración total: Se cumplió dicho principio en dos momentos; el primero al realizar las capacitaciones respecto a la distribución en planta, sus principios y su importancia, ya que se generó una integración total entre todas las áreas de la empresa para la comprensión de la importancia de todos en los procesos productivos. El segundo momento de cumplimiento de este principio fue generando equipos integrales en el área operativa para capacitarse en torno al funcionamiento de las diversas herramientas y máquinas que se tienen, ya sea para aprender a operarlas en un nivel básico, o bien, aprender a cómo reaccionar ante emergencias con las máquinas y herramientas, esto con el fin de promover la integración con las máquinas y materiales y saber las diversas situaciones que se pueden presentar.

Las capacitaciones se realizaron bajo el siguiente esquema:

Tabla 3. Cuadro de capacitaciones en torno a maquinaria en el equipo operativo

\begin{tabular}{|c|c|c|}
\hline Tema & Expositor & Equipo \\
\hline & & Juan Carlos Franco \\
Operación Básica de Centros & Cristina Arellano \\
Maquinado "CNC" & Marco Serratos \\
& Caciel Ruvalcaba \\
& Jordi Padilla & Cesar de la Rosa \\
flexis Ramselle \\
bmo actuar ante una emergencia en \\
un CNC o Torno? & & Abraham de la Cruz \\
& Alberto Díaz y Jordi Padilla & Marco Serratos \\
& & Edwin Ayala \\
& & Antonio Padilla \\
& & Dulce Castañeda \\
\hline
\end{tabular}




\begin{tabular}{|c|c|c|}
\hline & & $\begin{array}{c}\text { Caciel Ruvalcaba } \\
\text { Erick Montes } \\
\text { Antonio Acosta } \\
\text { Christopher Méndez }\end{array}$ \\
\hline & & Edwin Ayala \\
Manejo básico de la máquina de & & Antonio Padilla \\
grabado & Gustavo Ríos \\
& Erick Montes & Eusebio López \\
& & Caciel Ruvalcaba \\
& & Dulce Castañeda \\
& & Alejandro Negrete \\
& & Irving Monroy \\
\hline
\end{tabular}

Fuente: elaboración propia.

2. Principio de la mínima distancia: Tanto en almacén como en el área de acabados, se podría ver a simple vista que son espacios pequeños y siempre se tiene la mínima distancia, sin embargo, la naturaleza del proceso hace que en promedio se recorran $11 \mathrm{~m}$ y $18 \mathrm{~m}$ respectivamente en cada área entre cada proceso principal; en el área de almacén es surtimiento de pedidos y en el área de acabados es el proceso de pulido.

Se modificaron las ubicaciones de ciertas áreas de trabajo para cumplir con el principio de la mínima distancia, dichas modificaciones se mostrarán en los resultados al presentar la comparativa del layout antiguo con el actual.

3. Principio de recorrido: Las áreas de trabajo en la organización se encuentran ya acomodadas en función de los procesos, sin embargo, aún hay algunas mesas de trabajo y espacios específicos que no se encuentran alineadas al recorrido.

El principio de recorrido fue aplicado totalmente en el diseño de la nueva distribución del área de acabados, ya que era el área que más requería alinear el proceso y colocar de manera conjunta las áreas operativas. La nueva distribución del área de acabados se realizó gracias a que se pudo utilizar un área muerta de la empresa que no se requería para ningún proceso de valor, dicha área será mostrada en los resultados.

4. Principio de espacio cúbico: Este principio se enfoca principalmente en aprovechar los espacios verticales y horizontales de una o varias áreas para el almacenamiento de los productos tanto materias primas, en proceso como producto final, así como para la colocación de máquinas y herramientas para optimizar las áreas. Debido a que las máquinas y herramientas de las áreas de acabados son realmente pequeñas en tamaño, el principio de espacio cúbico fue implementado en su totalidad en el almacén de la empresa al utilizar un quinto y sexto nivel en racks para almacenar los productos de baja rotación y poder así, liberar los pasillos de material.

5. Principio de satisfacción y seguridad: La seguridad ha sido un factor fundamental en la empresa, por lo que, adquirido el compromiso de parte de los trabajadores con el proyecto de redistribución de áreas, también se logró el compromiso por parte de la empresa para dotar a los 
trabajadores de equipo e insumos para desarrollar sus tareas de manera adecuada. A todos los trabajadores se los proporcionaron botas de trabajo adecuadas a su medida, así como gafas y guantes de seguridad, sin mencionar que se colocaron al alcance caretas en caso de desempeñar alguna actividad que implique más riesgo.

En la fotografía siguiente se puede apreciar de los insumos que se proporcionaron a los empleados de la empresa.

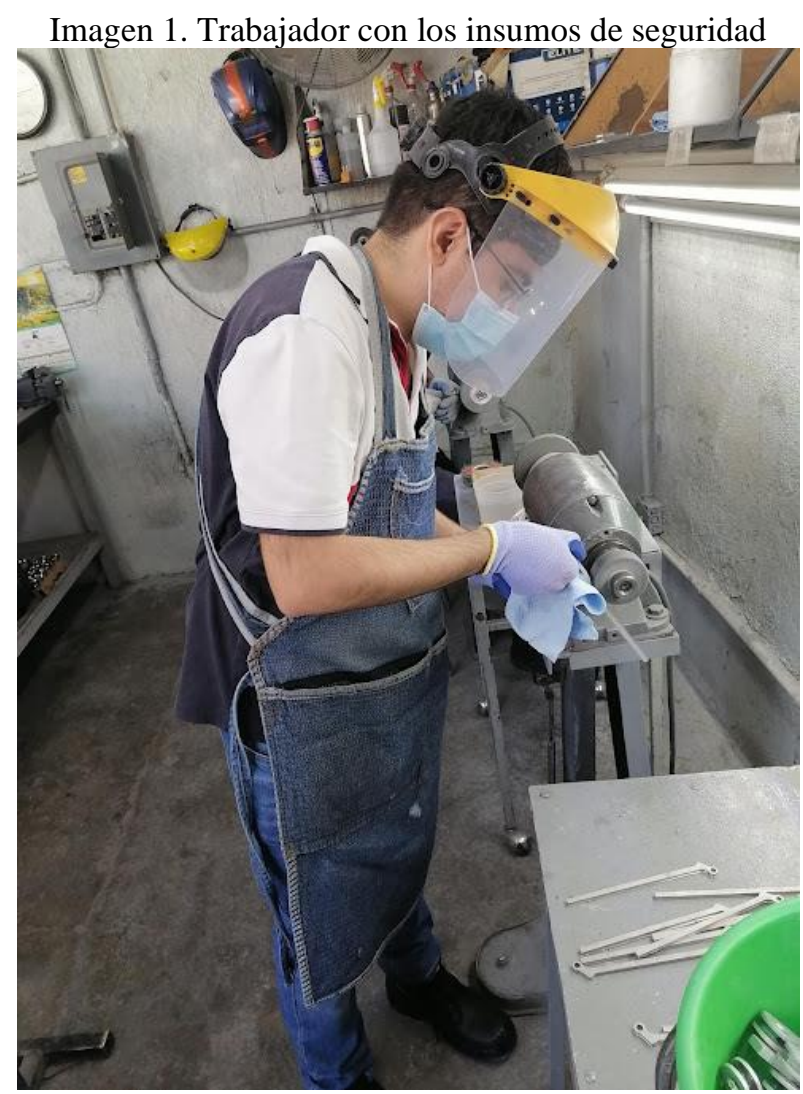

Fuente: Elaboración propia

La satisfacción de los empleados se logra mediante programas de revisión de desempeño con tres parámetros: revisión y cumplimiento de objetivos, desarrollo de nuevas habilidades y asignación y cumplimiento de tareas para ayudar a su crecimiento personal y profesional, con este tipo de herramientas se logra su satisfacción personal y organizacional. Por supuesto, que se tiene el compromiso de la empresa para cumplir las necesidades básicas de los trabajadores, así como el cuidado de su salud, en estos tiempos de pandemia, donde gracias a una buena gestión del cuidado de la salud se han evitado brotes internos y cabe resaltar que el $100 \%$ del personal se encuentra vacunado contra el SARS Cov-2. Se muestra a continuación el formato de evaluación de desempeño que se maneja en la empresa para la revisión de la satisfacción: 




Fuente: elaboración propia.

6. Principio de flexibilidad: Este principio es primordial, ya que permitirá ajustes en el futuro con la mínima interrupción en los procesos. La nueva distribución, permite que se realicen ajustes ya que las herramientas y mesas de trabajo tanto en el área de acabados como en el almacén se puede mover fácilmente en función de la necesidad y adaptarse a cambios rápidos que se requieran, esto es benéfico para la empresa ya que se manufacturan más de 90 tipos de productos y se necesitan maniobrar de distintas formas, por eso, el tener una distribución flexible permite a la organización ser más eficiente en la ejecución de los trabajos de producción en los tiempos requeridos. Lo anterior se puede confirmar con las siguientes imágenes:

Imagen 3. Área de acabados actual, herramientas y mesas flexibles.

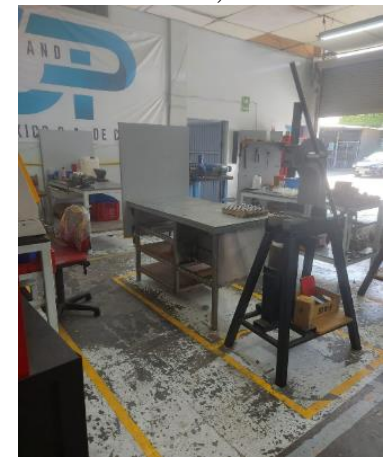

Fuente: elaboración propia. 


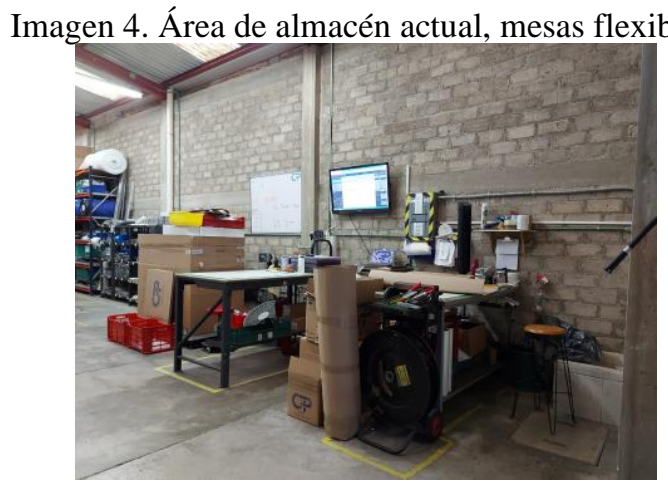

Fuente: elaboración propia.

\section{RESUMEN DE RESULTADOS}

La aplicación de los principios de la distribución en planta en las áreas de acabados y almacén en la empresa de producción de partes ortopédicas significó un aumento en la productividad la cual fue medida no solo en función de los tiempos de traslado y búsqueda de materiales, también en un un aumento del número de piezas obtenidas en el área de acabados. La tabla comparativa de tiempos es la siguiente:

Tabla 3. Comparación de tiempos de traslado y búsqueda de materiales en el área de acabados.

\begin{tabular}{|c|c|c|}
\hline Operación & Antes & Después \\
\hline $\begin{array}{c}\text { Búsqueda de } \\
\text { materia prima }\end{array}$ & $125 \mathrm{~s}$ & $\begin{array}{c}85 \mathrm{~s} \\
\text { *Disminuye }\end{array}$ \\
\hline $\begin{array}{c}\text { Transporte de } \\
\text { material a área } \\
\text { de proceso }\end{array}$ & $67 \mathrm{~s}$ & $\begin{array}{c}23 \mathrm{~s} \\
\text { *Disminuye }\end{array}$ \\
\hline $3 . \quad \begin{array}{c}\text { Transporte de } \\
\text { material a } \\
\text { almacén final }\end{array}$ & $17 \mathrm{~s}$ & $17 \mathrm{~s}$ \\
\hline
\end{tabular}

Fuente: elaboración propia

En el caso del almacén la comparativa fue más sencilla, pues solamente se cronometró una serie de 10 operaciones de surtimiento y se obtuvo el promedio, antes de la redistribución y después de la redistribución. Los resultados y las fotografías de los cambios son los siguientes:

Tabla 4. Comparación de tiempos de búsqueda y surtimiento de productos en el área de almacén.

\begin{tabular}{|c|c|c|}
\hline Operación & Antes (tiempo medio) & Después (tiempo medio) \\
\hline $\begin{array}{c}\text { Búsqueda y surtimiento de un } \\
\text { pedido con promedio de 4 a } 6 \\
\text { productos. }\end{array}$ & $302 \mathrm{~s}$ & $219 \mathrm{~s}$ \\
\hline
\end{tabular}

Fuente: elaboración propia. 
Fuente: elaboración propia.

Imágenes 7 y 8 . Almacén antes y después, insumos y materia prima.
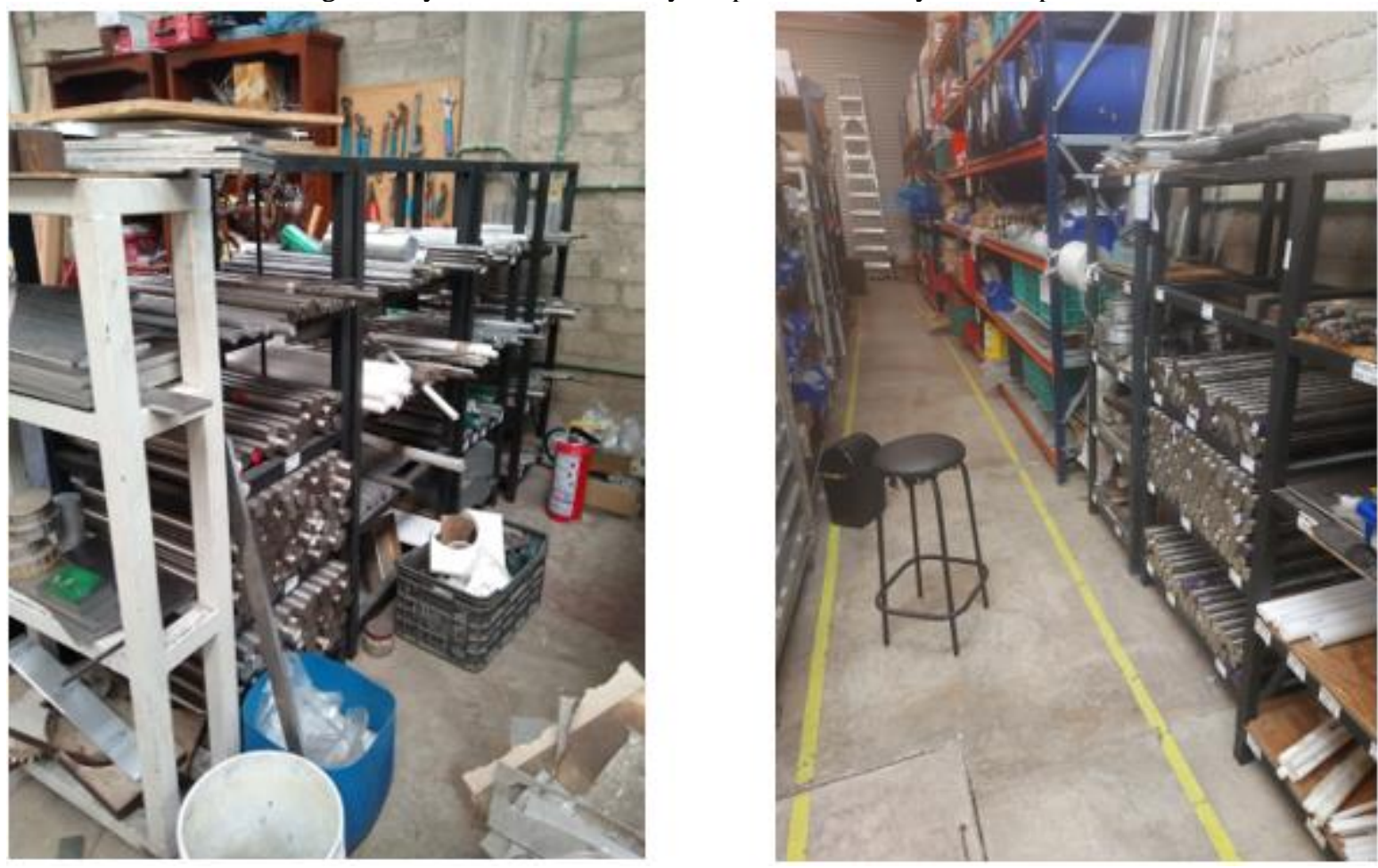

Fuente: elaboración propia.

Cómo podemos observar el cambio es notorio ya que se liberaron espacios de pasillos y en el área de rack se delimitaron los espacios para no revolver producto y tenerlo identificado con herramientas visuales.

Respecto a los layouts, aquí se muestra la redistribución que tuvieron las áreas de almacén y acabados respectivamente: 
Layouts 1 y 2. Distribución del área de acabados antes (izquierda) y distribución del área de acabados despúes (derecha).
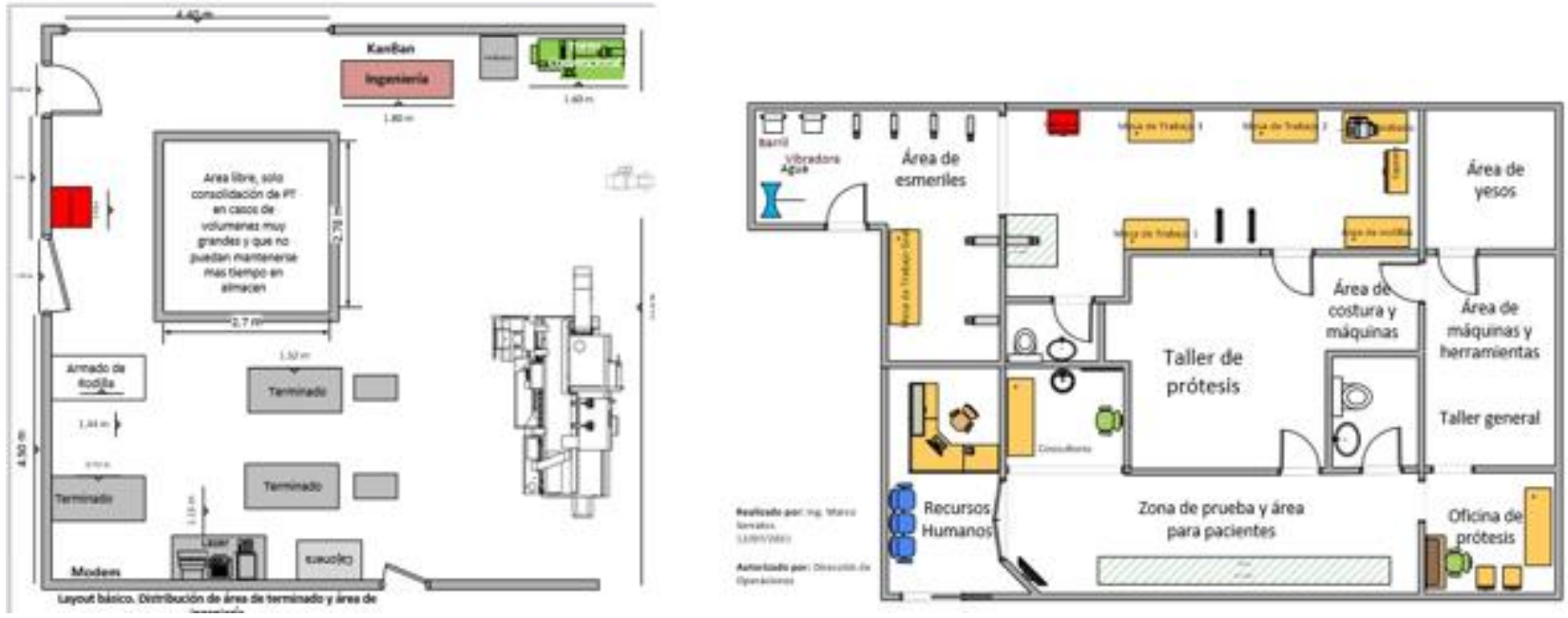

Fuente: elaboración propia.

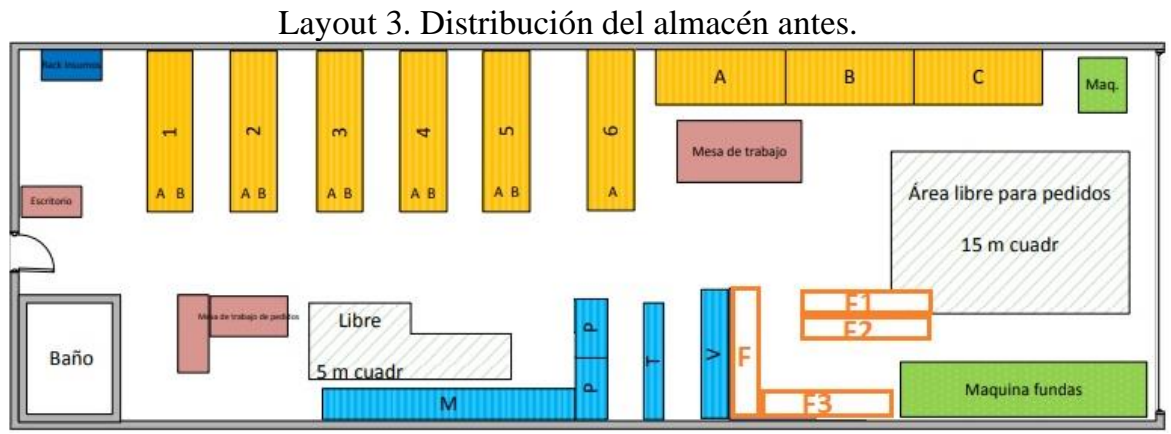

Fuente: elaboración propia.

Layout 4. Distribución del almacén después.

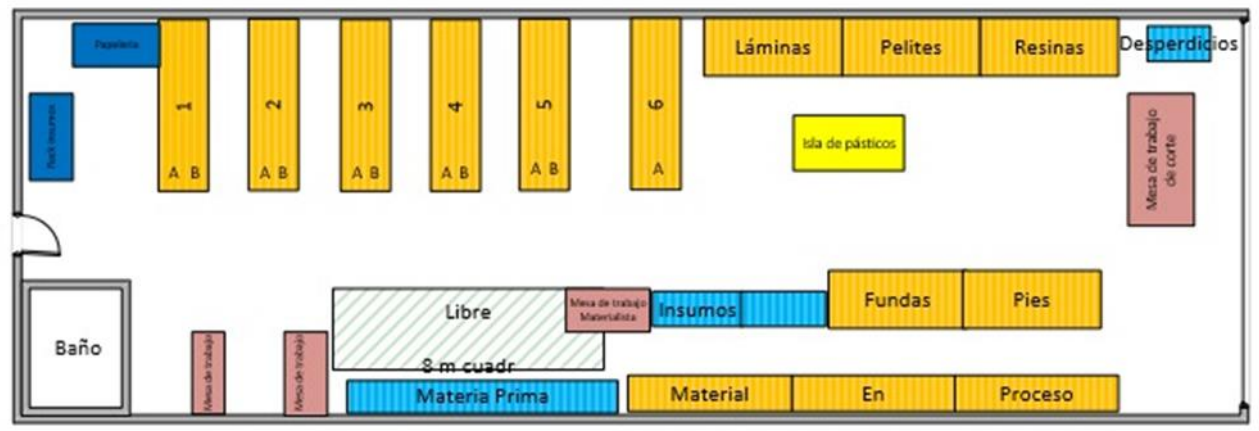

Fuente: elaboración propia.

\section{CONCLUSIONES}

La implementación de los principios de la distribución en planta ayuda indiscutiblemente a generar un aumento en la productividad de las operaciones de las áreas estudiadas en el presente documento, ya que en poco tiempo se pudo observar como las actividades fueron realizadas con mayor fluidez.

Parte del éxito fue el compromiso de los empleados y la alta dirección que pudieron generar ese 
cambio de mentalidad para conseguir los objetivos, una implementación de este tipo no se puede lograr sin el apoyo de todas las personas.

Con esta implementación se elimina la incertidumbre para cambios futuros y se deja de lado la cultura de "reacomodar" por hacerlo, pues de dejan las bases de que cuestiones y factores de deben tomar en cuenta para la distribución de las áreas.

La empresa productora de partes ortopédicas goza ahora de espacios seguros, ordenados y justificados en sus áreas gracias a los principios de la distribución en planta, los procesos productivos pueden desarrollarse ahora con mayor rapidez y exactitud. 


\section{REFERENCIAS}

Marcel Oswaldo Méndez-Mantuano, Salas Aplicación de la teoría de juegos para conocer el punto de equilibrio en las estrategias comerciales de dos empresas Vol. 3 No. 1 (2022): South Florida Journal of Development, Miami, v. 3, n. 1, jan./feb. 2022 Published: 2022-01-01

Muther R. (1982) Distribución en planta. Editorial: Hispano Europea S.A. Barcelona, España.

Muther R. (1982) Manejo de Materiales. Editorial: Hispano Europea S.A. Barcelona, España.

Platas G; Cervantes M. (2014) Planeación, diseño y layout. Grupo editorial Patria. Ciudad de México, México. 